\title{
Retorno à atividade produtiva de motociclistas vítimas de acidentes de trânsito
}

\author{
Motocyclists victims of traffic accidents return to normal productive activities \\ Retorno a la actividad productiva de los motociclistas víctimas de acidentes de tránsito
}

\section{Nelson Luiz Batista de Oliveira ${ }^{1}$, Regina Marcia Cardoso de Sousa ${ }^{2}$}

\begin{abstract}
RESUMO
Objetivos: caracterizar os motociclistas internados no hospital e residentes no município de Maringá, segundo as atividades desenvolvidas no momento do acidente, ocupação anterior ao trauma e o retorno à produtividade entre nove meses e um ano após esse evento, além de identificar os fatores associados para alteração da produtividade dessas vítimas. Métodos: a amostra foi constituída por 61 motociclistas vítimas de acidentes de trânsito nos meses de fevereiro e março de 1999. Os dados foram obtidos do prontuário hospitalar e de entrevistas domiciliares. Resultados: no momento do acidente 45,9\% das vítimas realizavam atividades relacionadas ao trabalho remunerado, enquanto $29,5 \%$ desenvolviam atividades de lazer, e as demais, outras atividades. Quanto à atividade produtiva das vítimas antes do acidente, o predomínio foi daquelas que realizavam atividade remunerada (81,9\%). No período entre nove e doze meses pós-trauma, 20,4\% das vítimas relataram atividade produtiva alterada. Conclusão: houve associação estatisticamente significativa entre a situação de produtividade após o acidente e as variáveis: Injury Severity Score, New Injury Severity Score e percepção do estado de saúde pela vítima.

Descritores: Acidentes de trânsito; Conseqüências de acidentes; Eficiência; Trabalho
\end{abstract}

\begin{abstract}
Objective: To characterize motorcyclists who have been victims of traffic accidents in Maringá, Paraná regarding their occupation, rehabilitation, or disability. Methods: A sample of 61 subjects participated in the study. Data were collected in February and March, 1999 though interviews and review of medical records. Results: Prior to the accident, the majority of subjects (45,9\%) were employed. A great number of them (45.9\%) were performing work-related activities in the moment of the accident. Among the remainder of victims, $29.5 \%$ were performing leisure activities. Nine to twelve months post accident, $20.4 \%$ of the victims had altered productive activities. Conclusion: There was a significant statistical association between productive activities and injury severity score, between productive activities and new injury severity score, and between productive activities and perception of health.
\end{abstract}

Keywords: Traffic accident; Accident complications; Efficiency; Work

\section{RESUMEN}

Objetivos: caracterizar a los motociclistas internados en el hospital y residentes en el municipio de Maringá, según las actividades desarrolladas en el momento del accidente, ocupación anterior al trauma y el retorno a la productividad entre nueve meses y un año después del evento además de identificar los factores asociados para la alteración de la productividad de esas víctimas. Métodos: la muestra estuvo constituida por 61 motociclistas víctimas de accidentes de tránsito en los meses de febrero y marzo de 1999. Los datos fueron obtenidos de la historia clínica hospitalaria y de entrevistas domiciliarias. Resultados: en el momento del accidente el $45.9 \%$ de las víctimas realizaban actividades relacionadas al trabajo remunerado, en cuanto que el 29.5\% desarrollaban actividades de recreación, y las demás, otras actividades. En cuanto a la actividad productiva de las víctimas antes del accidente, el predominio fue de aquellas que realizaban actividad remunerada (81.9\%). En el período entre nueve y doce meses post-trauma, el 20.4\% de las víctimas relataron actividad productiva alterada. Conclusión: hubo asociación estadísticamente significativa entre la situación de productividad después del accidente y las variables: Injury Severity Score, New Injury Severity Score y la percepción del estado de salud por la víctima.

Descritores: Accidentes de tránsito; Consecuencias de accidentes; Eficiencia; Trabajo

${ }^{1}$ Doutorando do Programa de Pós-Graduação em Enfermagem na Saúde do Adulto da Escola de Enfermagem da Universidade de São Paulo. Professor Assistente do Departamento de Enfermagem da Universidade Estadual de Maringá UEM - Maringá - (PR), Brasil.

${ }^{2}$ Professor Associado do Departamento de Enfermagem Médico-Cirúrgica da Escola de Enfermagem da Universidade de São Paulo USP - São Paulo (SP), Brasil. 


\section{INTRODUÇÃO}

Nas últimas décadas, as lesões provocadas por acidentes de trânsito têm-se convertido em uma das principais causas de morte e incapacidades em todo o mundo $^{(1)}$. O número de veículos em circulação, a desorganização do trânsito, a deficiência da fiscalização, as condições dos veículos, as imprudências dos usuários e a impunidade dos infratores contribuem significativamente para a ocorrência desses eventos na população ${ }^{(1-2)}$.

No Brasil o crescente aumento do número de veículos nas últimas décadas, em especial da frota de motocicletas como meio de trabalho, por ser ágil, econômico e de custo reduzido, também tem sido um fator que contribui para a manutenção das elevadas taxas de acidentes de trânsito $^{(1-3)}$.

A vulnerabilidade do usuário de moto tem sido demonstrada pelo número de acidentes envolvendo esse tipo de veículo. No impacto dos acidentes de motocicleta, com freqüência há ocorrência de um choque desigual com veículos de maior porte. Por outro lado, há que se considerar que o motociclista não tem a estrutura do veículo para protegê-lo, absorvendo toda a energia do impacto e sendo comumente projetado à distância.

Pelas próprias características do veículo, os motociclistas, muitas vezes jovens e em idade produtiva, estão constantemente propensos e sujeitos a adquirirem lesões que podem provocar a morte ou mesmo limitar, temporária ou definitivamente, o desenvolvimento de suas atividades diárias, com sério comprometimento no retorno a sua produtividade ${ }^{(2)}$.

O retorno à produtividade contribui, de forma decisiva para melhorar a integração social das vítimas de trauma, e é objetivo básico das ações de recuperação de um evento traumático, particularmente quando se considera o ônus individual e social do trauma ${ }^{(4-5)}$.

A atividade produtiva, além de proporcionar equilíbrio econômico, desempenha um papel fundamental no processo de reabilitação como fonte de satisfação pessoal e auto-estima, como elemento essencial para aquisição e manutenção das relações sociais e como componente fundamental para determinar o nível de influência do individuo entre os membros de seu grupo social ${ }^{(5)}$.

De modo geral, os estudos que analisam as causas externas, em seus principais aspectos epidemiológicos, abordam o problema sob o aspecto da morbimortalidade e/ou demanda dos serviços hospitalares, mas são escassas as referências quanto ao retorno à produtividade após o evento.

\section{OBJETIVOS}

Caracterizar os motociclistas vítimas de acidente de trânsito, segundo as atividades desenvolvidas no momento da ocorrência do evento, ocupação anterior ao trauma e retorno à produtividade, entre nove meses e um ano após o acidente.

Identificar os fatores associados à produtividade dos motociclistas após o trauma.

\section{MÉTODOS}

Trata-se de estudo descritivo correlacional com dados prospectivos e retrospectivos, no qual adotaram-se o referencial teórico e a metodologia de pesquisas que abordam a temática deste estudo ${ }^{(4-11)}$

A casuística de estudo foi constituída por motociclistas que se envolveram em acidentes de trânsito no município de Maringá, no Paraná, nos meses de fevereiro e março de 1999. Os critérios considerados para a seleção da amostra foram: internação hospitalar pós-trauma; idade acima de 14 anos; residência no município de Maringá; assistência pelo Sistema Integrado de Atendimento ao Trauma e Emergência (SIATE), cujo acionamento tenha gerado uma ficha de atendimento e o encaminhamento tenha sido feito a um dos hospitais de referência em trauma para a cidade de Maringá e região.

A fonte inicial de informações para localização das vítimas foi o Relatório de Atendimento do Socorrista (RAS). Esse relatório, preenchido pelo médico ou por outros profissionais da equipe de resgate do SIATE contém, além dos dados da vítima e do acidente, a indicação do hospital para o qual a mesma foi encaminhada.

No período de estudo foram identificadas, através dos registros do SIATE, 206 vítimas motociclistas, sendo 177 residentes em Maringá. Desse total de 177 vítimas, 124 foram atendidas em centros de referência para tratamento do trauma, 43 foram atendidas em outros hospitais e 10 recusaram atendimento pelo sistema de resgate. Das vítimas atendidas em centros de referência para atendimento ao trauma, 67 foram internadas.

Os dados relacionados aos objetivos desse trabalho foram coletados em prontuário hospitalar e por meio de entrevistas em visitas domiciliares, por meio de um questionário estruturado, realizadas com as vítimas que atendiam aos critérios de inclusão do estudo.

Para coleta dos dados constantes do prontuário hospitalar, foi elaborada uma ficha própria onde se registraram, conforme descrito nesses prontuários, os dados demográficos e clínicos da vítima, os diagnósticos das lesões, data de admissão e data da alta hospitalar. Posteriormente, todas as descrições dessas lesões foram utilizadas para realização do cálculo dos índices de gravidade do trauma: Injury Severity Score (ISS) e New Injury Severity Score (NISS) ${ }^{(12)}$.

Esses indicadores têm com base de cálculo a 
Abbreviated Injury Scale (AIS) que é um sistema de base eminentemente anatômica, apresentada sob a forma de manual, onde as lesões são listadas e a gravidade delas é indicada por uma escala numeral que varia de 1 , indicador de lesão leve, a 6 , de gravidade máxima ${ }^{(12)}$.

O ISS é definido pela aplicação de uma equação matemática que consiste na soma do quadrado do escore AIS mais elevado de três regiões do corpo ${ }^{(12)}$.

O NISS, índice derivado do ISS, proposto para corrigir algumas limitações do seu precursor e facilitar sua aplicação, consiste na soma dos quadrados dos escores AIS das três lesões mais graves, independente da região corpórea $^{(13)}$.

Os motociclistas internados nos hospitais de referência para atendimento do trauma, residentes na cidade de Maringá, foram localizados e entrevistados no domicílio, com a finalidade de obter dados sobre a atividade no momento do acidente, ocupação anterior ao trauma, retorno à produtividade e percepção do seu estado de saúde após o trauma.

As visitas domiciliares, realizadas entre nove meses e um ano após o trauma, foram feitas para 61 vítimas, das 67 inicialmente identificadas. Nessa fase, foram excluídas três vítimas que morreram na vigência da internação e outras três, não localizadas para a entrevista a domicílio. Durante a visita domiciliar, as seguintes condições das vítimas foram analisadas: autopercepção, orientação no tempo e lugar, capacidade de comunicação oral e escrita. Essas condições foram examinadas exclusivamente por meio da percepção do entrevistador.

Todos apresentaram condições para a entrevista, e aceitaram participar da pesquisa, assinando o Termo de Consentimento Livre e Esclarecido, de acordo com as diretrizes e normas regulamentadoras de pesquisas em seres humanos. A realização do estudo também foi formalmente autorizada pelo Corpo de Bombeiros SIATE, pelo Hospital Santa Casa, Hospital Santa Rita e pelo Comitê Permanente de Ética em Pesquisa da Universidade Estadual de Maringá.

O endereço e o telefone constante do prontuário e/ ou arquivos hospitalares foi o meio utilizado para localização das vítimas. O grupo de 61 vítimas foi visitado em suas residências no período de 5 de janeiro a 24 de fevereiro de 2000. Quando a vítima não era encontrada, agendava-se um novo retorno com um familiar próximo. No caso de não se encontrar nenhuma pessoa em casa, visitas eram feitas, tantas quantas fossem necessárias até se concretizar a entrevista.

Para análise dos dados sobre o retorno à produtividade, as vítimas foram agrupadas, de acordo com a seguinte categorização( ${ }^{(4)}$ :

- Atividade interrompida: indivíduos que após o trauma mantêm-se afastados da ocupação principal.

- Alterada devido ao trauma: indivíduos com mudança de ocupação conseqüente às condições pós-traumáticas; retorno à ocupação anterior sem conseguir o mesmo nível de desempenho; retorno à ocupação anterior reduzindo o tempo dedicado ao seu desempenho; desempregado após o trauma, porém mantendo trabalho remunerado exercido de forma irregular; estudantes e pessoas envolvidas em atividades do lar que retornaram a essas atividades sem conseguir manter o mesmo desempenho. São incluídos nesse grupo indivíduos que mantêm tanto ocupação remunerada como atividades escolares, casos afastados de uma dessas duas atividades ou, ainda, se numa delas ou ambas apresentar alteração de desempenho.

- Inalterada: indivíduos que retornaram a sua ocupação principal com o mesmo nível de desempenho.

Em relação à percepção do estado de saúde, as vítimas, conforme sua própria referência, foram categorizadas em estado de saúde melhor, inalterado e pior, sendo que no tratamento estatístico as duas primeiras categorias foram agrupadas.

Terminada a etapa de entrevistas e categorização das vítimas, os dados foram armazenados e analisados em um banco de dados computadorizado, construído com o programa EPI - INFO, versão 6.03 da Organização Mundial da Saúde.

Os dados estão apresentados em freqüência absoluta e relativa, mostrados em forma de tabelas e figuras. $\mathrm{O}$ Teste Exato de Fischer foi usado para verificar a associação entre a situação de produtividade com as seguintes variáveis: sexo, idade, escolaridade, ISS, NISS, presença de trauma crânio-encefálico (TCE), tempo de internação hospitalar, percepção da saúde e atividade remunerada. Em todas as análises, estabeleceu-se o nível de significância de 5\%.

\section{RESULTADOS}

Do grupo de 61 vítimas motociclistas 53 (86,8\%) eram do sexo masculino e $45(73,7 \%)$ eram jovens, sendo $21(34,4 \%)$ com idade $\geq 14$ e $<23$ anos e 24 $(39,3 \%)$ com idade $\geq 23$ e $<32$. Quanto ao tipo de acidente, predominou a colisão com $54(88,5 \%)$ casos, as quedas da motocicleta foram as causas externas das outras vítimas. A maior parte dos motociclistas, 51 $(83,6 \%)$, conduzia o veículo no momento do acidente, os demais estavam na condição de passageiro.

Das 61 vítimas analisadas, 17 (27,9\%) foram acometidas por TCE. A pontuação ISS e NISS $<16$ foram predominantes na amostra, 93,4\% e 91,8\%, respectivamente. O tempo de internação dessas vítimas variou de 1 a 10 dias, média de 2,20 (DP=2,09) e a escolaridade foi em média 4,18 (DP=1,69). A escolaridade variou entre ensino fundamental incompleto e nível superior completo. 


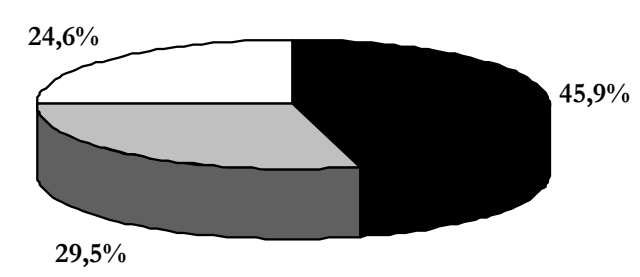

Atividades relacionadas ao trabalho remunerado $\square$ Atividade de Lazer

$\square$ Outras Atividades

Figura 1: Motociclistas vítimas de acidente de trânsito, segundo atividade desenvolvida no momento do acidente. Maringá - PR, $1999 / 2000$.

Os dados apresentados na Figura 1 mostram que, $28(45,9 \%)$ vítimas motociclistas, realizavam atividades relacionadas ao trabalho remunerado, no momento do acidente. Das demais vítimas, 18 (29,5\%) estavam em atividades de lazer e $15(24,6 \%)$ realizavam outras atividades. Nesta última categoria foram incluídas as vítimas que realizavam atividades que atendiam necessidades pessoais tais como: transporte de familiares, deslocamento para compras e pagamentos, atividades religiosas ou residência de familiares.

Do total de 28 vítimas que no momento do acidente estavam em atividades relacionadas ao trabalho, 19 $(67,8 \%)$ estavam trabalhando e $9(32,1 \%)$ estavam no trajeto do trabalho. Das 19 vítimas que estavam trabalhando no momento do acidente $10(52,6 \%)$ eram entregadores e os demais exerciam atividades variadas tais como: cobrador, mecânico, vendedor, repositor, divulgador, vidraceiro, entre outras.
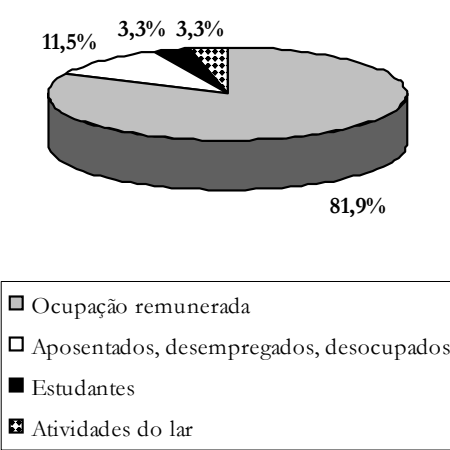

Figura 2: Motociclistas vítimas de acidente de trânsito segundo ocupação principal antes do trauma. Maringá - PR, 1999/2000.

Os dados apresentados na Figura 2 mostram que no período anterior ao acidente, $81,9 \%$ das vítimas, exerciam alguma atividade remunerada, $11,5 \%$ eram aposentados, desempregados ou desocupados. Estudantes e pessoas que exerciam atividades do lar somaram $3,3 \%$ cada.

Tabela 1: Motociclistas vítimas de acidente de trânsito, segundo situação de produtividade entre nove e doze meses pós-trauma

\begin{tabular}{lcc}
\hline SITUAÇÃO DE PRODUTIVIDADE & $\mathbf{N}^{\mathbf{*}} *$ & $\mathbf{\%}$ \\
\hline Atividade interrompida & - & - \\
Alterada devido ao trauma & 11 & 20,4 \\
Inalterada & 43 & 79,6 \\
\hline & & \\
TOTAL & $\mathbf{5 4}$ & $\mathbf{1 0 0 , 0}$ \\
\hline
\end{tabular}

* Exclui 7 aposentados, desempregados, desocupados

Os dados apresentados na Tabela 1 mostram que todas as vítimas motociclistas retornaram à produtividade entre nove meses e um ano após o acidente, no entanto 11 (20,4\%), relataram alterações na atividade produtiva.

Os dados da Tabela 2 permitem observar associações estatisticamente significativas entre a situação de produtividade após o acidente e as variáveis: ISS, NISS e percepção da saúde. A maioria das vítimas que apresentou alteração na produtividade informou piora na percepção do seu estado de saúde $(72,7 \%)$, quando comparado ao período anterior ao evento traumático. A presença de TCE, não esteve associada à alteração na situação de produtividade.

\section{DISCUSSÃO}

Nos últimos anos, o baixo custo para aquisição e manutenção da motocicleta, a má distribuição de renda e o agravamento das condições socioeconômicas, colaboram para a maior utilização desse veículo em nosso país ${ }^{(3)}$. Assim, as motocicletas, usadas por um grande número de pessoas, contribuem para o aumento da frota circulante e o envolvimento de motociclistas em acidentes ${ }^{(1)}$.

$\mathrm{O}$ acidente de trânsito é um evento complexo que envolve falhas humanas, dos veículos, além dos fatores desfavoráveis ambientais. Muitas causas destes acidentes envolvem manobras arriscadas no manejo do veículo, uso de álcool e drogas, velocidade excessiva, cansaço, influências climáticas, vias e sinalização inadequadas ou falta de manutenção dos veículos. Os motociclistas que trabalham no trânsito têm como fator coadjuvante dessas causas de acidentes, as longas jornadas de trabalho no trânsito intenso das grandes cidades, além das pressões e as cobranças relacionadas ao aumento de produtividade $^{(1)}$.

Os dados deste estudo mostram que a maior parte dos indivíduos no momento do acidente estava envolvida em atividades de trabalho, portanto favorecem as indicações de que o uso freqüente das motocicletas 
Tabela 2 - Teste Exato de Fischer e valores de $p$ para as variáveis demográficas e clínicas, segundo a situação de produtividade entre nove e doze meses dos motociclistas* vitimas de acidente de trânsito.

\begin{tabular}{|c|c|c|c|c|c|c|}
\hline \multirow[t]{3}{*}{ Variáveis } & \multirow[t]{3}{*}{ Categorias } & \multicolumn{4}{|c|}{ Situação de Produtividade } & \multirow[t]{3}{*}{$\mathrm{p}$} \\
\hline & & \multicolumn{2}{|c|}{ Alterada } & \multicolumn{2}{|c|}{ Inalterada } & \\
\hline & & $\mathbf{N}^{\mathbf{o}}$ & $\%$ & $\mathbf{N}^{\circ}$ & $\%$ & \\
\hline \multirow[t]{2}{*}{ Sexo } & Feminino & 2 & 18,18 & 4 & 9,30 & 0,5900 \\
\hline & Masculino & 9 & 81,82 & 39 & 90,70 & \\
\hline \multirow[t]{2}{*}{ Idade (anos) } & $\geq 14<30$ & 6 & 54,54 & 29 & 67,44 & 0,4895 \\
\hline & $\geq 30 \leq 60$ & 5 & 45,46 & 14 & 32,56 & \\
\hline \multirow[t]{2}{*}{ Escolaridade } & $<8$ anos & 6 & 54,54 & 19 & 44,19 & 0,5386 \\
\hline & $\geq 8$ anos & 5 & 45,46 & 24 & 55,81 & \\
\hline \multirow[t]{2}{*}{ ISS } & $<16$ & 8 & 72,72 & 42 & 97,68 & 0,0234 \\
\hline & $\geq 16$ & 3 & 27,28 & 1 & 2,32 & \\
\hline \multirow[t]{2}{*}{ NISS } & $\overline{<} 16$ & 7 & 63,63 & 42 & 97,68 & 0,0046 \\
\hline & $\geq 16$ & 4 & 36,37 & 1 & 2,32 & \\
\hline \multirow[t]{2}{*}{ TCE } & Sim & 3 & 27,28 & 11 & 25,59 & 1,0000 \\
\hline & Não & 8 & 72,72 & 32 & 74,41 & \\
\hline \multirow[t]{2}{*}{ Tempo de internação } & $<5$ dias & 8 & 72,72 & 39 & 90,69 & 0,1404 \\
\hline & $\geq 5$ dias & 3 & 27,28 & 4 & 9,31 & \\
\hline \multirow[t]{2}{*}{ Percepção da saúde } & Melhor/Inalterada & 3 & 27,27 & 42 & 97,67 & $<0,0001$ \\
\hline & Pior & 8 & 72,73 & 1 & 2,33 & \\
\hline \multirow[t]{2}{*}{ Atividade remunerada } & $\operatorname{Sim}$ & 11 & 100,0 & 39 & 90,69 & 0,5707 \\
\hline & Não & - & - & 4 & 9,31 & \\
\hline
\end{tabular}

* Excludos 7 aposentados, desempregados, desocupados.

no trabalho contribui para o aumento dos acidentes com esse tipo de veículo.

Por outro lado, as atividades de lazer, outra freqüente atividade no momento dos acidentes estudados, são propícias ao consumo de álcool e até de drogas ilícitas. Soma-se a isto, os percursos não habituais percorridos para desenvolver essas atividades e quando ocorrem nos finais de semana podem estar relacionados com o aumento da velocidade média dos veículos circulantes.

$\mathrm{Na}$ época do evento traumático, a grande maioria das vítimas, apresentava ocupação remunerada e encontravase na faixa etária de 14 a 35 anos. Assim como neste estudo, o predomínio de jovens em idade produtiva é observado em pesquisas, quando vítimas de acidentes de trânsito são caracterizadas. $\mathrm{O}$ adulto jovem apresenta-se como vítima freqüente de trauma, fato que ocasiona, não só um dano social, mas perdas na população economicamente ativa ${ }^{(14)}$.

O retorno à produtividade é freqüentemente base para medida de recuperação de um trauma ou doença e é particularmente relevante quando se examina o ônus individual ou social do trauma, em que a maioria dos atingidos é o adulto jovem. Dessa forma, uma das principais metas de recuperação consiste em retornar o indivíduo à sua atividade anterior, isto é, retorno ao emprego, à escola ou às atividades domésticas ou outra atividade relevante ${ }^{(0)}$.

No presente estudo, todas as vítimas, no período entre 9 e 12 meses pós-trauma, haviam retornado à sua ocupação principal, porém 20,4\% delas, ainda relatavam alteração na produtividade em conseqüência do trauma.
Poucos estudos na literatura têm analisado o retorno à produtividade de vítimas de trauma em geral. $\mathrm{O}$ mais freqüente tem sido avaliar retorno à atividade remunerada ou a análise de grupos específicos de vítimas, tais como, aquelas que apresentam TCE ou trauma grave.

Pesquisadores que analisam conseqüências do trauma em vítimas de TCE estimam que o retorno à produtividade dessas vítimas varia entre $20 \%$ e $50 \%$, dependendo da gravidade da lesão, ocupação anterior, características demográficas e suporte ambiental e familiar $^{(7-8)}$.

Estudo realizado em hospital de referência para atendimento de trauma, na cidade de São Paulo, com vítimas de TCE de todas gravidades, mostrou que, no período de um ano, 63,9\% dos participantes do estudo haviam retornado à produtividade com o mesmo desempenho; $19,4 \%$ tinham alteração em conseqüência ao trauma e $16,7 \%$ encontravam-se inativos ${ }^{(9)}$.

A presença de TCE é fator agravante do prognóstico da vítima de trauma, tanto quando se analisa mortalidade, como morbidade pós-traumática. Na pesquisa atual, a ausência de inativos no período de aproximadamente um ano pós-trauma aponta para resultado mais favorável que o observado no estudo realizado em São Paulo ${ }^{(9)}$ com vítimas de TCE, e parece condizente com as indicações apresentadas na literatura. No entanto, associação entre produtividade alterada e presença de TCE não foi constatada no grupo analisado.

No presente estudo, o ISS, o NISS e a percepção do estado de saúde pela vítima foram fatores associados com a situação produtiva dos motociclistas, cerca de 12 meses 
pós-trauma.

Estudo desenvolvido na Grã Bretanha com vítimas atendidas pelo sistema aéreo de resgate mostrou que, aos 12 meses pós-trauma, o pleno retorno ao trabalho foi de $60,8 \%$. O retorno, com alterações nas atividades de trabalho ocorreu em apenas $8,4 \%$ dos casos, porém 30,8\% das vítimas eram incapazes de retornar à sua atividade remunerada. Nesse estudo, também foi observada diferença estatisticamente significativa no retorno ao trabalho, entre aqueles com ISS $<16$ e ISS $\geq 16$. O retorno ao trabalho de forma inalterada foi de $81,1 \%$, entre vítimas com ISS $<16$ e de 39,1\%, entre aquelas com ISS $\geq 16^{(10)}$.

Em relação à percepção do estado de saúde, pesquisa realizada com 247 vítimas de diferentes mecanismos de trauma, sem neurotrauma grave, mostra que 64\% dessas vítimas haviam retornado ao trabalho no período de 12 meses após o trauma ${ }^{(11)}$.

Para finalizar, convém lembrar que a amostra foi de vítimas sobreviventes pelo menos nove meses pós-trauma. A exclusão de indivíduos que morreram antes da entrevista domiciliar minimizou a gravidade das vítimas estudadas, além do que, as características de planejamento urbano da cidade de Maringá pode ter contribuído para os resultados relacionados a essa variável. O número de casos relativamente reduzido, outra limitação deste estudo, requereu a formação de categorias amplas para as análises de associação, perdendo-se parcialmente a especificidade da informação coletada.

\section{CONCLUSÃO}

O estudo evidencia o freqüente uso da motocicleta como veículo para atividades de trabalho, reafirma a importância dos acidentes de trânsito entre jovens em idade produtiva e o comprometimento da produtividade de muitas vítimas por longos períodos, após o evento traumático. Sugere a importância da regulamentação e fiscalização das atividades de trabalho realizadas pelos motociclistas, visando minimizar a magnitude dos acidentes com esse tipo de veículo e aumentar a segurança, nas vias públicas, dos motociclistas e demais usuários.

Os resultados obtidos também revelaram especificidades importantes, relacionadas ao retorno à produtividade dessas vítimas após o acidente, as quais podem contribuir para a melhoria da assistência e implementação de políticas e estratégias de redução dos acidentes de motocicleta.

O retorno à produtividade, indicador importante de resultados do trauma para a vítima e a sociedade, é influenciado por vários fatores, sendo evidenciada, no atual estudo, a gravidade do trauma, indicada pelo ISS e NISS, e a percepção do estado de saúde da pessoa traumatizada.

\section{REFERÊNCIAS}

1. Bastos YGL, Andrade SM, Soares DA. Características dos acidentes de trânsito e das vítimas atendidas em serviço pré-hospitalar em cidade do Sul do Brasil, 1997/2000. Cad Saúde Pública. 2005; 21(3): 815-22.

2. Oliveira NLB, Sousa RMC. Diagnóstico de lesões e qualidade de vida de motociclistas, vítimas de acidentes de trânsito. Rev Latinoam Enfermagem. 2003: 11(6): 749-56.

3. Liberatti CLB, Andrade SM, Soares DA, Matsuo T. Uso de capacete por vítimas de acidentes de motocicleta em Londrina, sul do Brasil. Rev Panam Salud Publica. 2003; 13(1): 33-8.

4. Sousa RMC, Koizumi MS. As vítimas de traumatismo crânio-encefálico seis meses após o trauma. Acta Paul Enfermagem. 1998; 11(1): 63-78.

5. Muñoz-Céspedes JM, Paul-Lapedriza NM, Pelegrín-Valero C, Tirapu-Ustarroz J. Factores de pronóstico em los traumatismos craneoencefálicos. Rev Neurol. 2001; 32(4): 351-64.

6. MacKenzie EJ, Siegel JH, Shapiro S, Moody M, Smith RT. Functional recovery and medical costs of trauma: an analysis by type and severity of injury. J Trauma. 1988; 28(3): 281-97.

7. Wehman P, Kregel J, Keyser-Macus L, Sherron-Targett P, Campbell L, West M, Cifu DX. Supported employment for persons with traumatic brain injury: a preliminary investigation of long-term follow-up costs and program efficiency. Arch Phys Med Rehabil. 2003; 84(2): 192-6.

8. Keyser-Marcus LA, Bricout JC, Wehman P, Campbell LR, Cifu DX, Englander J, et al. Acute predictors of return to employment after traumatic brain injury: a longitudinal follow-up. Arch Phys Med Rehabil. 2002; 83(5): 635-41.

9. Sousa RMC, Koizumi MS. Vítimas de trauma crânioencefálico e seu retorno à produtividade após 6 meses e 1 ano. Rev Esc Enfermagem USP. 1999; 33(3): 313-22.

10. Baldry Currens JA Evaluation of disability and handicap following injury. Injury. 2000; 31(2): 99-106.

11. Michaels AJ, Michaels CE, Smith JS, Moon CH, Peterson C, Long WB. Outcome from injury: general health, work status, and satisfaction 12 months after trauma. J Trauma. 2000; 48(5): 841-8; discussion 848-50.

12. Association for the Advancement of Automotive Medicine - AAAM. The Abbreviated Injury Scale (AIS): 1990 revision. Update 1998. Des Plaines, IL; 1998.

13. Tay SY, Sloan EP, Zun L, Zaret P. Comparison of the New Injury Severity Score and the Injury Severity Score. J Trauma. 2004; 56(1): 162-4.

14. Brasil. Ministério da Saúde. Portaria MS/GM n 737 de 16/05/01. Política Nacional de Redução da Morbimortalidade por acidentes e violências. Diário Oficial da República Federativa do Brasil, Brasília (DF); 2001 Maio 18; no 96, Seção 1e. 